\title{
Prospecção científica e tecnológica com ênfase na atividade anticâncer do óleo essencial de Aeschynomene denticulata
}

Scientific and technological prospection with emphasis on the anticancer activity of the essential oil of Aeschynomene denticulata

Prospección científica y tecnológica con énfasis en la actividad anticancerígena del aceite esencial de Aeschynomene denticulata

Rodrigo Elísio de Sá

ORCID: https://orcid.org/0000-0002-1492-3995 Universidade Federal do Delta do Parnaíba, Brasil E-mail: rodrigosa238@gmail.com

Gisele Santos Araújo

ORCID: https://orcid.org/0000-0002-1756-6823 Universidade Federal do Delta do Parnaíba, Brasil E-mail: giselearaujo92@gmail.com

Paulo Sérgio de Araujo Sousa

ORCID: https://orcid.org/0000-0001-8764-4455 Universidade Federal do Delta do Parnaíba, Brasil

E-mail: psergio.araujosousa @gmail.com

Dakson Douglas Araújo

ORCID: https://orcid.org/0000-0003-0102-7240 Universidade Federal do Delta do Parnaíba, Brasi E-mail: dacksondouglas@gmail.com

Fabrício dos Santos Machado

ORCID: https://orcid.org/0000-0003-1637-5934

Universidade Federal do Delta do Parnaíba, Brasil E-mail: fabricio.machado10@live.com

Lucicleia Dias Monteiro

ORCID: https://orcid.org/0000-0002-6904-4787

Universidade Federal do Delta do Parnaíba, Brasil E-mail: Lucicleiabiomed@gmail.com

Jaiane Cruz dos Santos

ORCID: https://orcid.org/0000-0002-4939-2565

Universidade Federal do Delta do Parnaíba, Brasil

E-mail: jaiane.cruz30@gmail.com

Valentina Rhémily de Melo Vasconcelos

ORCID: https://orcid.org/0000-0002-3735-9936

Universidade Federal do Delta do Parnaíba, Brasil

E-mail: valentinarhemily23@ hotmail.com

Gabriella Linhares de Andrade

ORCID: https://orcid.org/0000-0003-2481-9646

Universidade Federal do Delta do Parnaíba, Brasil E-mail: gabriellalinhares9@gmail.com

Gabrielle Costa Sousa

ORCID: https://orcid.org/0000-0001-8496-3477 E-mail: gabygabryelle19@hotmail.com Universidade Federal do Delta do Parnaíba, Brasil

Lucas Florencia da Silva

ORCID: https://orcid.org/0000-0002-7575-3643

E-mail: lucasflorenci@gmail.com

Universidade Federal do Delta do Parnaíba, Brasil

\section{Resumo}

O câncer é uma doença caracterizada pela multiplicação e proliferação descontrolada de células geneticamente modificadas, sendo uma das principais causas de morte no mundo. A terapia disponível até o presente momento é o uso de quimioterápicos que apresentam efeitos adversos aos pacientes, e, além disso, são inespecíficos pois afetam células normais. Desse modo, a busca por informações sobre moléculas com atividade anticâncer torna-se uma ferramenta relevante para o mapeamento e aplicações biotecnológicas. Assim, objetivou-se realizar uma prospecção 
científica e tecnológica da planta Aeschynomene denticulata com ênfase no óleo essencial e sua atividade anticâncer em artigos e patentes indexadas em plataformas nacionais e internacionais. Para isso, foram utilizadas palavras-chave e combinações dos termos com o operador booleano AND para a busca: "Aeschynomene denticulata", "Antitumor", "Cancer", "Essential oil" e "Metastasis". Os resultados demonstram que boa parte dos artigos e patentes se detém ao descritor Aeschynomene denticulata. Nas bases científicas, PubMed, Scielo e Scopus apresentaram respectivamente 3, 7 e 23 artigos. Nas bases tecnológicas a única a apresentar patentes foi a EPO, num total de 23 trabalhos, considerando a Alemanha em destaque na proteção da pesquisa envolvendo Aeschynomene denticulata. Quando utilizados em combinação dos descritores não foram encontrados artigos e/ou patentes. Dessa forma, conclui-se que poucos são os estudos e tecnologias desenvolvidas com a espécie vegetal e inexistentes são os trabalhos envolvendo aplicações do óleo essencial no câncer.

Palavras-chave: Fabaceae; Câncer; Óleo essencial.

\begin{abstract}
Cancer is a disease characterized by the uncontrolled multiplication and proliferation of genetically modified cells, being one of the main causes of death in the world. The therapy available to date is the use of chemotherapeutics that have adverse effects on patients, and, in addition, are non-specific as they affect normal cells. Thus, the search for information about molecules with anticancer activity becomes a relevant tool for mapping and biotechnological applications. Thus, the objective was to carry out a scientific and technological survey of the plant Aeschynomene denticulata with emphasis on essential oil and its anticancer activity in articles and patents indexed in national and international platforms. For this, keywords and combinations of terms with the Boolean operator AND were used for the search: "Aeschynomene denticulata", "Antitumor", "Cancer", "Essential oil" and "Metastasis". The results show that most of the articles and patents use the descriptor Aeschynomene denticulata. In the scientific databases, PubMed, Scielo and Scopus presented 3, 7 and 23 articles, respectively. In terms of technology, the only one to present patents was EPO, in a total of 23 works, considering Germany highlighted in the protection of research involving Aeschynomene denticulata. When used in combination of descriptors, no articles and/or patents were found. Thus, it is concluded that there are few studies and technologies developed with the plant species and that there are no works involving applications of essential oil in cancer.
\end{abstract}

Keywords: Fabaceae; Cancer; Essential oil.

\title{
Resumen
}

El cáncer es una enfermedad caracterizada por la multiplicación y proliferación incontrolada de células modificadas genéticamente, siendo una de las principales causas de muerte en el mundo. La terapia disponible hasta la fecha es el uso de quimioterápicos que tienen efectos adversos en los pacientes y, además, no son específicos ya que afectan a las células normales. Así, la búsqueda de información sobre moléculas con actividad anticancerígena se convierte en una herramienta relevante para el mapeo y aplicaciones biotecnológicas. Así, el objetivo fue realizar un relevamiento científico y tecnológico de la planta Aeschynomene denticulata con énfasis en el aceite esencial y su actividad anticancerígena en artículos y patentes indexados en plataformas nacionales e internacionales. Para ello, se utilizaron palabras clave y combinaciones de términos con el operador booleano AND para la búsqueda: "Aeschynomene denticulata", "Antitumoral", "Cáncer", "Aceite esencial” y "Metástasis". Los resultados muestran que la mayoría de los artículos y patentes utilizan el descriptor Aeschynomene denticulata. En las bases de datos científicas, PubMed, Scielo y Scopus presentaron 3, 7 y 23 artículos, respectivamente. En cuanto a tecnología, la única en registrar patentes fue EPO, en un total de 23 trabajos, considerando a Alemania destacada en la protección de las investigaciones que involucran a Aeschynomene denticulata. Cuando se usa en combinación de descriptores, no se encontraron artículos ni patentes. Así, se concluye que existen pocos estudios y tecnologías desarrolladas con las especies vegetales y que no existen trabajos que involucren aplicaciones del aceite esencial en el cáncer.

Palabras clave: Fabaceae; Cáncer; Aceite esencial.

\section{Introdução}

O câncer é uma doença caracterizada pela multiplicação e proliferação descontrolada de células geneticamente modificadas, sendo uma das principais causas de morte no mundo. A complexidade patológica do câncer leva a alternativas terapêuticas muitas vezes usadas em associação visando combater a carcinogênese. Entretanto, nem sempre se tem obtido resultados satisfatórios, pois, observando-se elevadas taxas de recidiva do tumor e o aparecimento de metástases, além da indução de muitos efeitos colaterais (Weinberg, 2011; Silva et al., 2020).

As características do câncer compreendem diferentes capacidades biológicas adquiridas durante o desenvolvimento do tumor. Esse tipo de neoplasia consiste em várias etapas complexas que incluem sustentar a sinalização proliferativa, evitar os 
supressores de crescimento, resistir à morte celular, possibilitar a imortalidade replicativa, induzir angiogênese, reprogram ação do metabolismo energético, fuga à destruição imune e metástase. Além disso, o recrutamento de células normais do organismo contribui para aquisição de características marcantes ao criar o microambiente tumoral (Weinberg, 2011)

Mundialmente, o câncer é considerado uma das principais causas de morte e os números apresentados são alarmantes. A estimativa global do câncer é grande, o que representou 8,8 milhões de mortes em 2015 e deve aumentar até 14 milhões até 2030. Cerca de $63 \%$ dos casos das mortes ocorrem em países em desenvolvimento, fato este relacionado com as causas da doença e dificuldades no seu tratamento (Abbas; Rehman, 2018; Sakthivel; Guruvayoorappan, 2018).

A metástase é a principal razão para a mortalidade resultante de pacientes com essa patologia, sendo um fator importante na definição do prognóstico de pacientes com câncer (INCA, 2017). Novos estudos buscam constantemente diferentes estratégias a fim de combater a progressão tumoral e aperfeiçoar o tratamento dos pacientes. Vários estudos das diversas características das células cancerosas podem trazer resultados para o desenvolvimento de drogas alvo direcionadas (Baudino, 2015).

Por sua vez, os produtos naturais são base na formação dos produtos terapêuticos. A extensa diversidade química desses compostos permite várias atividades biológicas por meio de diversos mecanismos, permitindo a elas, por exemplo, atividade citotóxica e antiproliferativa. Várias drogas anticâncer são descritas sendo derivadas de produtos naturais. Dentre elas, está o cloridrato de irinotecano, docetaxel, valrubicina e fosfato de etoposideo, que foram aprovadas pelo Food and Drug Administration (FDA) (Newman; Cragg, 2016).

Neste contexto, as plantas do gênero Aeschynomene possuem potencial farmacológico, como mostra um levantamento bibliográfico realizado por Fullas e colaboradores (1996), em que algumas espécies desse gênero foram estudadas com relação aos aspectos farmacológicos, etnofarmacológicos e composição química, revelando sua importância na produção de compostos com propriedades antiinflamatória, analgésica, citotóxica, anti-herpética e efeito depressor do sistema nervoso central (SNC).

A espécie Aeschynomene denticulata (Fabaceae), popularmente conhecida como "corticeirinha", "anguiquinho" ou "paquinha", é uma planta nativa do Brasil, sendo um subarbusto ou erva invasora que ocorre principalmente em locais úmidos, florescendo de novembro a janeiro. No Brasil, essa espécie é amplamente difundida, com registros no Norte (Amazonas e Pará), Nordeste (Bahia e Pernambuco), Centro-Oeste (Goiás, Mato Grosso do Sul e Mato Grosso), Sudeste (Minas Gerais, Rio de Janeiro e São Paulo) e Sul (Paraná, Santa Catarina e Rio Grande do Sul), sendo presente em vários biomas tais como Floresta Amazônica, Caatinga, Cerrado, Mata Atlântica, Pampa e Pantanal (Maia et al., 2015).

Desde os tempos antigos, as plantas aromáticas são utilizadas por seu valor medicinal. Os óleos essenciais derivados dessas plantas podem ser usados como alternativas/adjuvantes eficazes em aplicações farmacêuticas e biomédicas (Sharma et al., 2019). Assim, nesse seguimento, cabe destacar que diferentes mecanismos podem ser responsáveis pelos efeitos citotóxicos relatados de óleos essenciais ou seus constituintes. Estes incluem indução de morte celular por apoptose e/ou necrose, parada do ciclo celular e perda da função de organelas-chave, dentre outras. Alguns desses efeitos são atribuíveis à natureza lipofílica e aos baixos pesos moleculares dos constituintes dos óleos essenciais, que lhes permitem atravessar as membranas celulares alterando as camadas de fosfolipídios, aumentando a fluidez da membrana e levando ao vazamento de íons e do conteúdo citoplasmático (Wei; Shibamoto, 2010; Tuttolomondo et al., 2013).

Assim sendo, o mapeamento científico e tecnológico de pesquisas em relação a atividade anticâncer do óleo essencial de $A$. denticulata tornam-se relevantes para que a identificação das aplicações científicas e tecnológicas, desenvolvidas pela espécie, sejam encontradas e assim permita mapear sistematicamente o estudo no intuito de propor uma aplicação inovadora em prol de amparar as pesquisas nos moldes da farmacologia oncológica.

Desse modo, este artigo objetivou identificar os principais estudos e aplicações tecnológicas desenvolvidas com a espécie A. denticulata, com ênfase no seu óleo essencial e na atividade anticâncer, em plataformas de publicações de artigos e 
patentes nacionais e internacionais.

\section{Metodologia}

O mapeamento envolvendo a planta A. denticulata ocorreu por meio de pesquisas em sites nacionais e internacionais de publicação de artigos e patentes, a saber: Scientific Electronic Library Online (SciELO), PubMed, Scopus e Web of Science $^{\mathrm{TM}}$, para artigos, e Instituto Nacional de Propriedade Industrial do Brasil (INPI), European Patent Office (EPO), World Intellectual Property Organization (WIPO) e United States Patent and Trademark Office (USPTO), para as patentes. Para as investigações, realizadas durante o período de 01 de novembro 2020 a 31 de agosto de 2021, foram utilizados os registros encontrados nas bases até a data da realização da prospecção, em que considerou-se os documentos que apresentarem no título e/ou no resumo as seguintes palavras-chave: Aeschynomene denticulata, Aeschynomene denticulata and antitumor, Aeschynomene denticulata and cancer, Aeschynomene denticulata and essential oil and metastasis, Aeschynomene denticulata and essential oil and cancer e Aeschynomene denticulata and essential oil and antitumor (Rocha et al., 2020; Sousa et al., 2021).

Para a busca dos materiais científicos foi utilizado como critério de inclusão, artigos que apresentavam correlações com a temática abordada e/ou aqueles que explorassem os mecanismos biológicos da Aeschynomene denticulata associadas ao câncer. Portanto, estudos desse cunho são importantes pois abrem perspectivas para novas pesquisas e contribui para o desenvolvimento tecnológico e científico (Sousa et al., 2021).

Como critério de exclusão dos artigos no trabalho, foram considerados apenas aqueles que apresentavam correlações com o tema de pesquisa e/ou que mostravam potencial anticâncer da espécie A. denticulata. Ademais, para os dados obtidos com a prospecção científica dos artigos, considerou-se o conteúdo científico abordado e ano de publicação do trabalho acadêmico, enquanto que para as patentes depositadas, avaliou-se a Classificação Internacional de Patentes (CIP), ano e país/organização de depósito (Lima et al., 2015).

\section{Resultados e Discussão}

Os resultados da prospecção científica e tecnológica em bases nacionais e internacionais permite amparar novas pesquisas, principalmente no eixo inovação tecnológica e ineditismo. Portanto, através dessas análises com as palavras-chave para as buscas, foi possível traçar um perfil da temática proposta, afim de elucidar estudos já desenvolvidos até o presente momento e patentes. Os dados desta pesquisa são apresentados e discutidos a seguir.

\subsection{Prospecção Científica}

Assim sendo, as buscas para as bases de publicações de artigos do Scopus, SciELO, PubMed e Web of Science ${ }^{\mathrm{TM}}$ reportaram pouquíssimos artigos indexados para as palavras-chaves utilizadas. Os dados referentes as quantidades de artigos para cada base encontram-se elencados a seguir, na Tabela 01. 
Tabela 1 - Quantidades de artigos identificadas nas buscas e suas respectivas porcentagens.

\begin{tabular}{ccccccccc}
\hline & \multicolumn{2}{c}{ SciELO } & \multicolumn{2}{c}{ PubMed } & \multicolumn{2}{c}{ Scopus } & \multicolumn{2}{c}{ Web of Science $^{\text {TM }}$} \\
\hline Palavras-chave & $\mathbf{Q T}^{+}$ & \% & QT & \% & QT & \% & QT & \% \\
\hline $\begin{array}{c}\text { Aeschynomene denticulata } \\
\text { Aeschynomene denticulata } \text { and } \\
\text { Antitumor }\end{array}$ & - & 100 & 3 & 100 & 12 & 100 & 0 & 0 \\
\hline $\begin{array}{c}\text { Aeschynomene denticulata } \text { and } \\
\text { Cancer }\end{array}$ & - & - & - & - & - & - & - & - \\
\hline $\begin{array}{c}\text { Aeschynomene denticulata } \text { and } \\
\text { Essential oil and Metastasis }\end{array}$ & - & - & - & - & - & - & - & - \\
\hline $\begin{array}{c}\text { Aeschynomene denticulata } \text { and } \\
\text { Essential oil and Cancer }\end{array}$ & - & - & - & - & - & - & - & - \\
\hline $\begin{array}{c}\text { Aeschynomene denticulata } \text { and } \\
\text { Essential oil and Antitumor }\end{array}$ & - & - & - & - & - & - & - & - \\
\hline
\end{tabular}

Fonte: Autores (2021).

Nota: +QT é utilizado na tabela como a abreviação da palavra "Quantidade” nesta tabela e "_"é utilizado como um indicativo de que não houve nenhum resultado retornado para a busca.

Em relação aos resultados, nota-se uma baixa quantidade de artigos encontrados, porém ao analisar uma certa quantidade deles, foi possível perceber que vários se repetem nas diversas bases de dados pesquisadas. Os artigos científicos são um veículo de divulgação de conhecimento científico (Mueller; Perucchi, 2014) e, a partir do baixo número de artigos encontrados nessas bases de dados, reafirma-se a necessidade do incentivo para a produção de novos trabalhos, pois a busca de literatura científica como meio de estudo e de desenvolvimento leva o leitor a investigação através de novos estudos, o que impulsionaria a busca por trabalhos relacionados ao câncer e novas formas de tratamento dessa doença (Kirchhof; Lacerda, 2012).

Desde 1950, agentes antitumorais têm sido desenvolvidos a partir de plantas e até hoje os produtos naturais ainda representam uma importante fonte de novas moléculas bioativas e promissoras contra várias doenças. Uma vez que $80 \%$ da população mundial depende da medicina tradicional para suas necessidades básicas de saúde e quase $85 \%$ dessa tradição envolve o uso de plantas medicinais e seus princípios ativos (Andricopulo et al., 2009). Nas últimas décadas, o retorno à terapêutica natural trouxe de volta os fitoterápicos para as farmácias de todo o país, entretanto o conhecimento de seu uso e suas aplicações é pouco, ou é variavelmente confuso. Com isso, os óleos essenciais tem se apresentado como uma excelente fonte de compostos com propriedades anticâncer, o que acaba impulsionando a procura, bem como a divulgação de artigos científicos nesse eixo temático.

Dessa forma, os resultados da busca científica na base do SciELO para a palavra-chave "Aeschynomene denticulata" reportaram 7 artigos publicados, no PubMed 3 artigos e no Scopus 12 trabalhos.

Já para o mesmo termo, a base de publicações Web of Science ${ }^{\mathrm{TM}}$ não retornou nenhum resultado para a busca. No entanto, no PubMed encontraram-se apenas três artigos, onde um apresentou repetição com outros abordados em outras plataformas. Logo, apresentou-se em um estudo a anatomia foliar de sete espécies anfíbias do gênero Aeschynomene, dentre essas a A. denticulata (Leme; Scremin-Dias, 2014). Em outro estudo avaliou a atividade acaricida in vitro de extratos de 21 espécies de plantas do Pantanal mato-grossense, incluindo a espécie de angiquinho (Santos et al.,2013). 
Figura 2 - Distribuição de publicações de artigos, por ano, envolvendo a palavra-chave Aeschynomene denticulata na base SciELO.

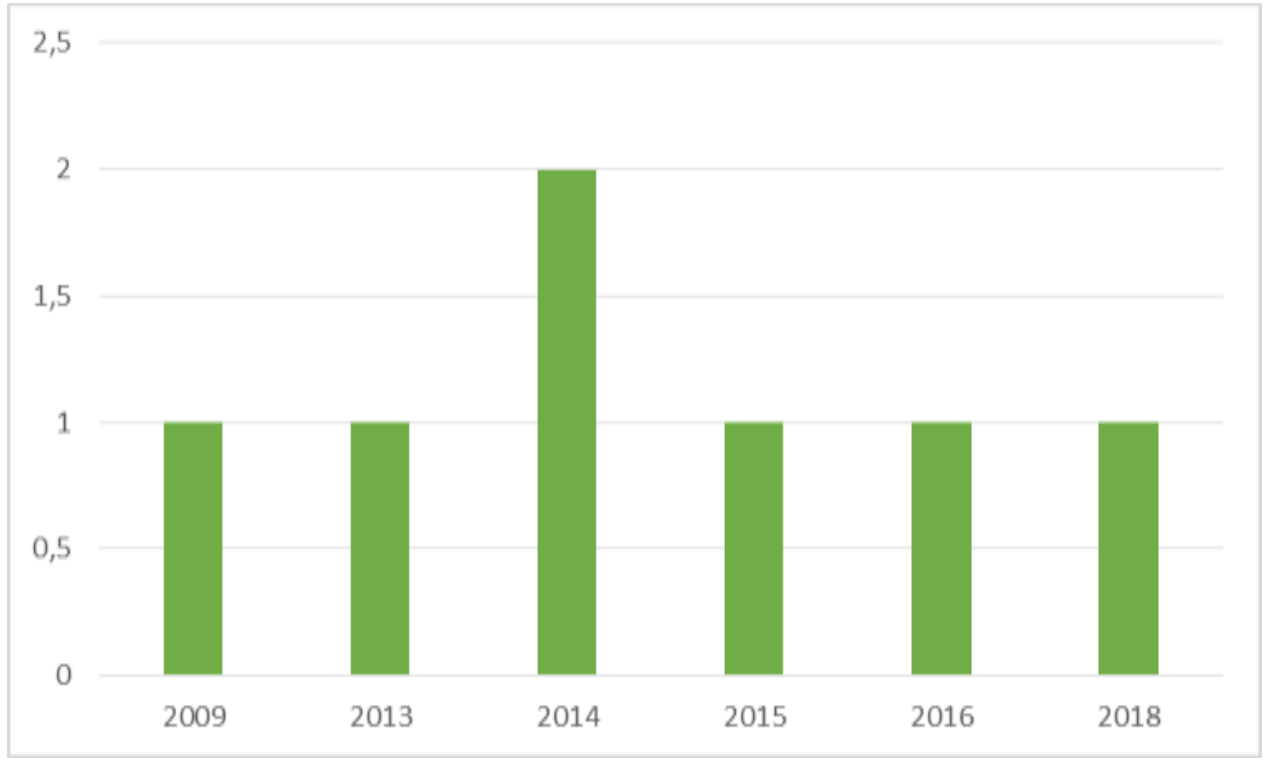

Fonte: Autores (2021).

Dentre os artigos encontrados com a busca para "Aeschynomene denticulata" na SciELO, identificou-se que um artigo aborda a identificação e quantificação das principais espécies de plantas daninhas presentes no arroz irrigado, sendo entre essa a conhecida popularmente como angiquinho (Nunes; Schaedler; Chiapinotto, 2018). Outro estudo avaliou a habilidade competitiva relativa de cultivares de arroz na presença de um biótipo de angiquinho (A. denticulata) em diferentes proporções substitutivas de plantas na associação (Galon et al., 2015). Os demais estudos encontrados no SciELo também abrangem essa interação da espécie em estudo nas plantações de arroz.

Figura 3 - Distribuição de publicações de artigos, por ano, envolvendo a palavra-chave Aeschynomene denticulata na base Scopus.

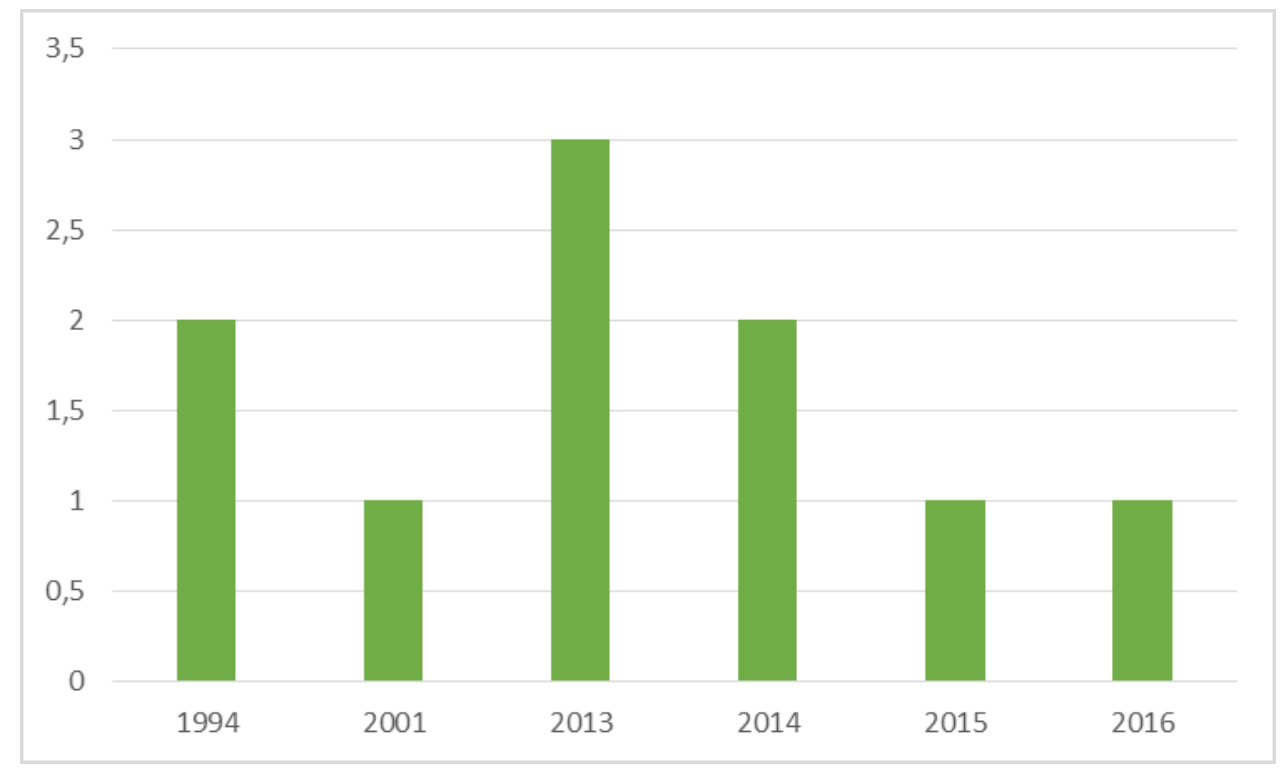

Fonte: Autores (2021). 
No que concerne aos resultados com o termo "Aeschynomene denticulata" para a base de artigos Scopus, a mesma retornou um total de 12 artigos entre os anos de 1994 e 2018, sendo que o ano que recebeu a maior quantidade de artigos publicados foi 2013. Além disso, os artigos encontrados nesta busca reportaram, majoritariamente, estudos com as investigações de interferência do angiquinho nas plantações de arroz. Isso demonstra que apesar de ser a base científica com quantidade de artigos, todos se restringiam ao eixo da agriculta. Logo, observa-se a necessidade de explorar a espécie, principalmente em estudos farmacológicos.

Dessa maneira, as buscas para as combinações das palavras-chave nas bases do SciELO, PubMed e Web of Science ${ }^{\mathrm{TM}}$ não retornaram artigos publicados (Tabela 01), indicando que inexistem trabalhos com as atividades anticâncer, antitumoral e antimetastática do óleo essencial da espécie A. denticulata.

\subsection{Prospecção Tecnológica}

No tocante as buscas para as patentes nas bases nacionais e internacionais, as pesquisas mostraram pouquíssimas tecnologias e/ou processos relacionados a espécie vegetal Aeschynomene denticulata. Em relação as combinações de palavras não foram identificadas nenhuma patente, como está sumarizado na Tabela 2.

Tabela 2 - Quantidades de patentes identificadas nas buscas e suas respectivas porcentagens.

\begin{tabular}{|c|c|c|c|c|c|c|c|c|c|c|}
\hline \multirow[b]{2}{*}{ Palavras-chave } & \multicolumn{2}{|c|}{ INPI } & \multicolumn{2}{|c|}{ LATIPAT } & \multicolumn{2}{|c|}{ EPO } & \multicolumn{2}{|c|}{ WIPO } & \multicolumn{2}{|c|}{ USPTO } \\
\hline & $\mathbf{Q T}^{+}$ & $\%$ & QT & $\%$ & QT & $\%$ & QT & $\%$ & QT & $\%$ \\
\hline Aeschynomene denticulata & - & - & - & - & 23 & 100 & - & - & - & - \\
\hline $\begin{array}{c}\text { Aeschynomene denticulata } \\
\text { and } \\
\text { Antitumor }\end{array}$ & - & - & - & - & - & - & - & - & - & - \\
\hline $\begin{array}{c}\text { Aeschynomene denticulata } \\
\text { and Cancer }\end{array}$ & - & - & - & - & - & - & - & - & - & - \\
\hline $\begin{array}{l}\text { Aeschynomene denticulata } \\
\text { and } \\
\text { Essential oil and Metastasis }\end{array}$ & - & - & - & - & - & - & - & - & - & - \\
\hline $\begin{array}{c}\text { Aeschynomene denticulata } \\
\text { and } \\
\text { Essential oil and Cancer }\end{array}$ & - & - & - & - & - & - & - & - & - & - \\
\hline $\begin{array}{l}\text { Aeschynomene denticulata } \\
\text { and } \\
\text { Essential oil and Antitumor }\end{array}$ & - & - & - & - & - & - & - & - & - & - \\
\hline
\end{tabular}

Nota: +QT é utilizado para abreviar a palavra "Quantidade” nesta tabela e “-“é utilizado como um indicativo de que não houve nenhum resultado retornado para a busca.

Fonte: Autores (2021).

Em relação aos dados tecnológicos envolvendo a planta $A$. denticulata, as bases do INPI, WIPO e USPTO não retornaram nenhum resultado para os termos empregados nas buscas. Entretanto, a base do EPO apresentou registros de patentes depositadas quando aplicado o descritor "Aeschynomene denticulata".

Ao utilizar-se o buscador "Aeschynomene denticulata" na base do EPO, obteve-se um total de 23 documentos de patentes retornados, depositados entre os anos de 2007 e 2020, sendo que os anos de 2019 e 2020 foram os que receberam as maiores quantidades de depósitos de patentes, com $48 \%$ e $39 \%$ respectivamente dos depósitos totais, mostrado na figura 4. 
Figura 4 - Depósito de patentes, de acordo com a CIP, para o termo "Aeschynomene denticulata" na base de depósito do EPO.

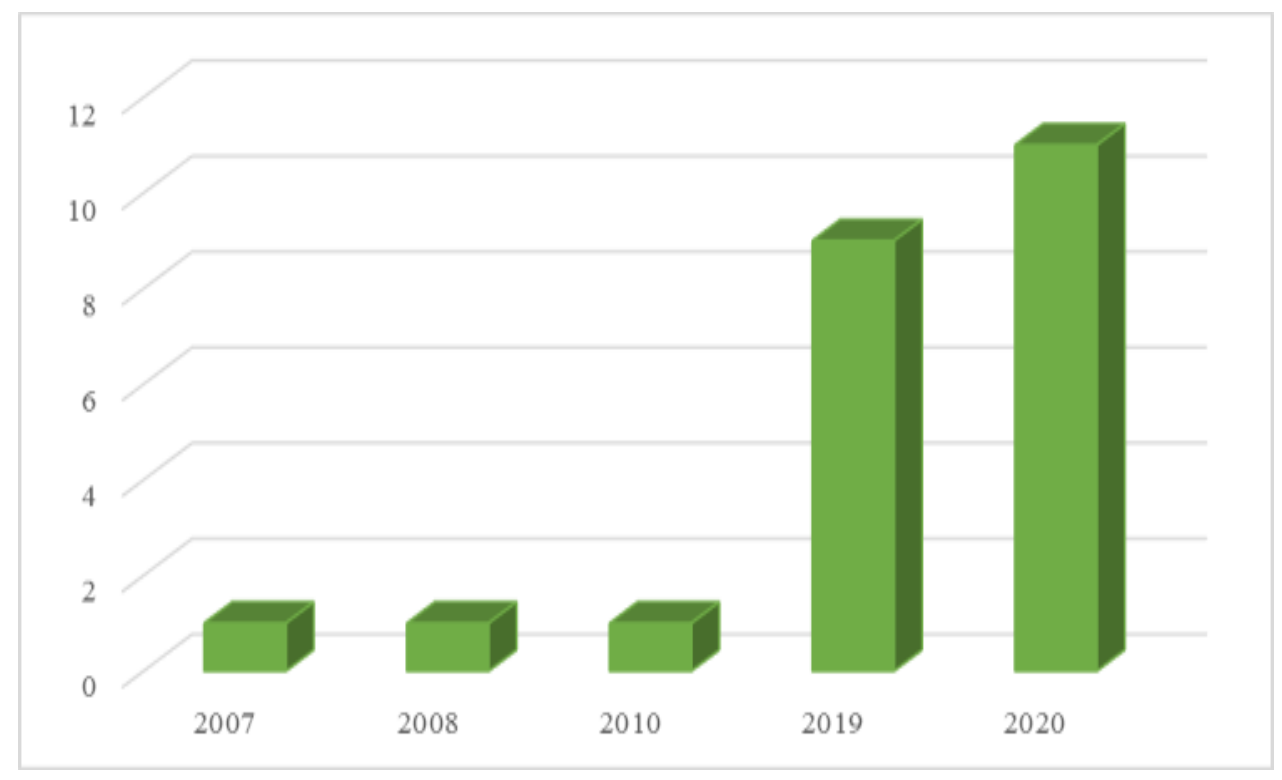

Fonte: Autores (2021).

Pode-se associar o elevado desenvolvimento de tecnologias nos anos de 2019 e 2020 envolvendo plantas do gênero Aeschynomene relacionado a resistência que estas plantas apresentam a herbicidas em plantações de arroz e a depreciação que as sementes de espécies do gênero Aeschynomene podem ocasionar em grãos de arroz, gerando assim ônus para os produtores agrícolas (Kissmann; Groth, 2000; Galon et al., 2015).

Além disso, ainda para a pesquisa com o termo "Aeschynomene denticulata" na base do EPO, pode-se identificar que os países que realizaram os depósitos destas tecnologias foram a Alemanha (74\%), Japão (22\%) e Suíça (4\%) (Figura 6), em que a Alemanha possui a maioria das tecnologias desenvolvidas sobre o seu domínio.

Em relação as temáticas das patentes encontradas na presente busca, pode-se identificar que as mesmas estavam voltadas principalmente para o desenvolvimento de novas misturas de herbicidas para cultura o controle de espécies de ervas daninhas que podem prejudicar a produção de determinadas culturas agrícolas, como, por exemplo, a produção de arroz, algodão e soja (WO2019030091A2; US2020359630A1). No entanto, não foi identificado nenhuma patente que fosse voltada para o uso do óleo essencial de "Aeschynomene denticulata" e seu efeito associado a terapia oncológica. 
Figura 5 - Distribuição dos países depositantes envolvendo a palavra-chave "Aeschynomene denticulata”, na base EPO.

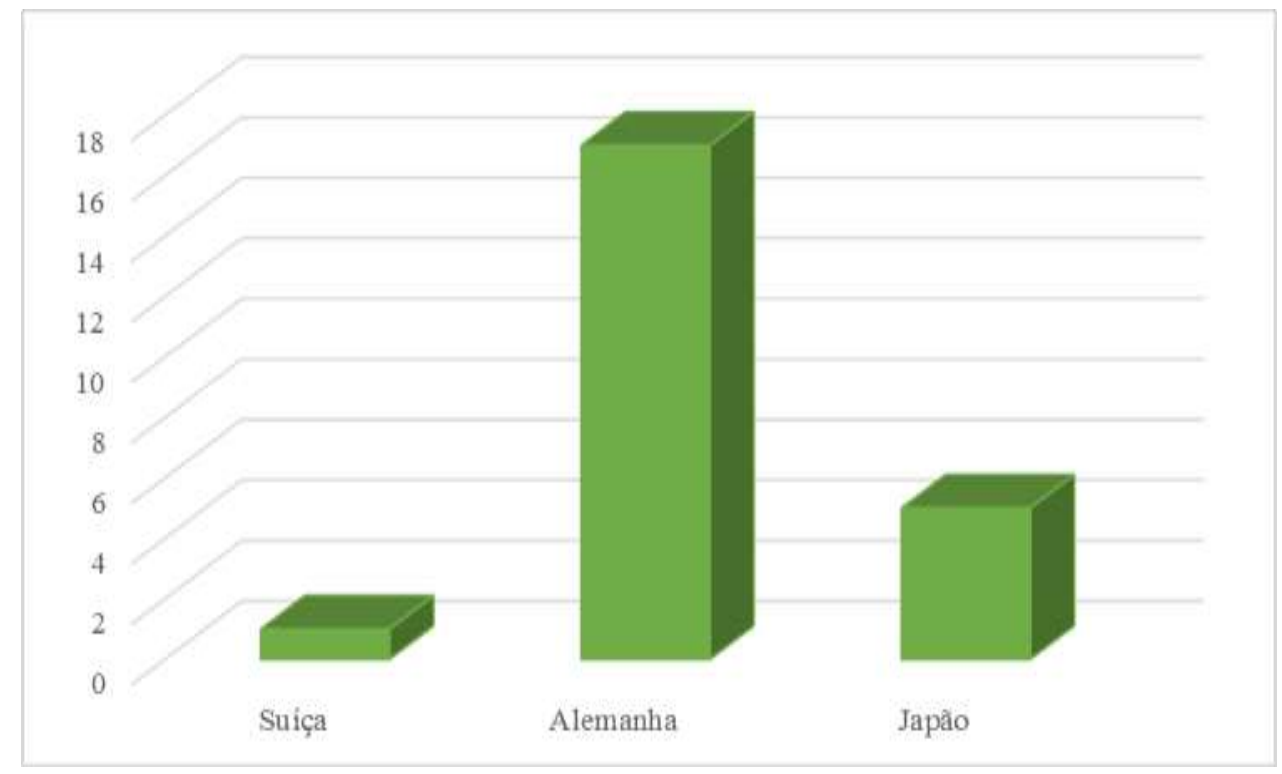

Fonte: Autores (2021).

Figura 6 - Distribuição de depósitos de patentes por ano e Porcentagem da distribuição dos países depositantes envolvendo a palavra-chave "Aeschynomene denticulata", na base EPO.

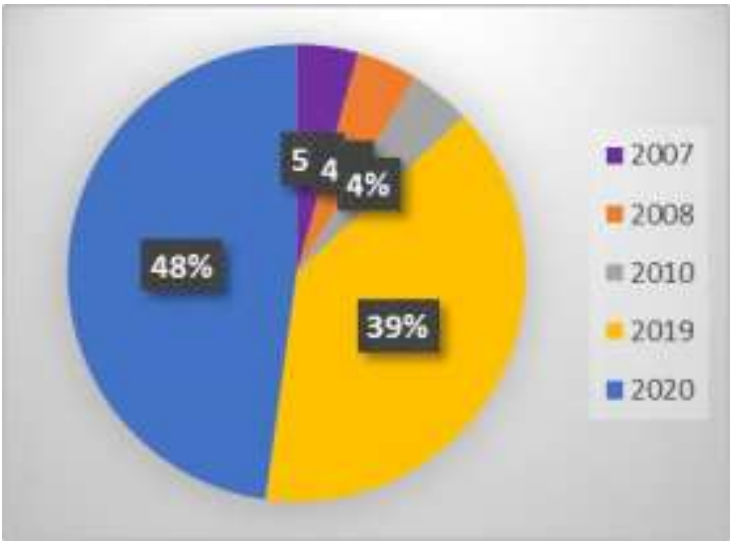

Fonte: Autores (2021).

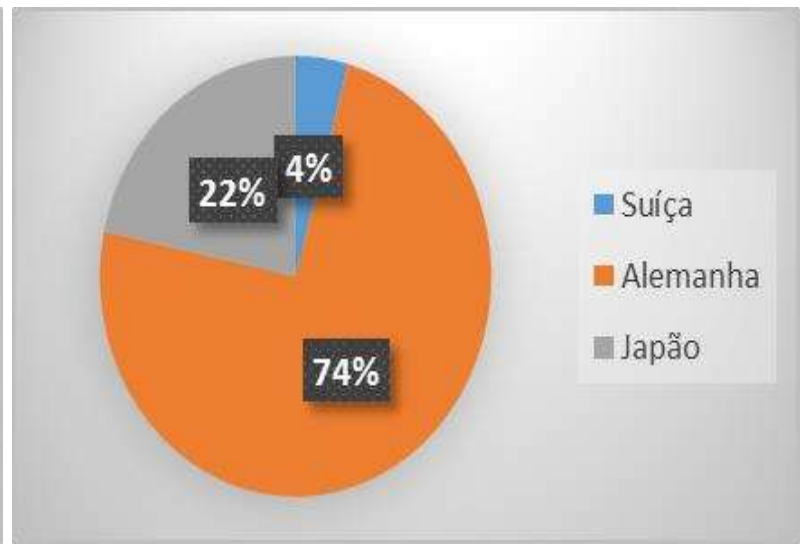

Em se tratando das combinações de palavras-chave envolvendo as bases de depósitos de patentes nacionais e internacionais abordadas na pesquisa, nenhuma patente foi reportada para as buscas dos descritores cruzados (tabela 02), indicando que são inexistentes as tecnologias desenvolvidas com o óleo essencial do angiquinho para o combate dos processos correlacionados com o câncer.

Logo, as pesquisas realizadas nos sites de depósitos de patentes do INPI, LATIPAT, EPO, WIPO e USPTO retornaram poucas tecnologias patenteadas relacionadas a inovação com o angiquinho. Observa-se que o Brasil, mesmo apresentando a espécie $A$. denticulata em vários estados não possui publicações de patentes com a referida espécie. Isso evidencia a necessidade de se buscar aproveitar as moléculas presentes na espécie, bem como suas aplicações biotecnológicas. 


\section{Considerações Finais}

Por meio desta prospecção nas bases de publicações de artigos do SciELO, PubMed e Web of Science ${ }^{\mathrm{TM}}$ e Scopus encontrou-se a existência moderada de artigos publicados relacionados a palavras-chave "Aeschynomene denticulata". Entretanto, os estudos se detêm a estudar a espécie filogeneticamente, bem como a sua interferência nas plantações de rizicultura. Logo, nota-se que tanto os moldes de óleo essencial e aplicação no câncer ainda é algo inexplorado.

Em se tratando das buscas nas bases de patentes do INPI, LATIPAT, EPO, WIPO e USPTO, as pesquisas demonstraram que as poucas patentes depositadas envolvem o termo "Aeschynomene denticulata", sendo que estas tratam, principalmente, do gênero Aeschynomene e seu potencial herbicida. Desse modo, ainda pode-se destacar que nenhuma patente mostrando a aplicação tanto de óleo essencial, como também de propriedades biológicas são identificáveis, o que torna o presente estudo inovador e inédito no eixo experimental. Além disso, observou-se também que os principais países e organizações depositantes de patentes são Alemanha (74\%), Japão (22\%) e Suíça (4\%) e a EPO.

Dessa forma, conclui-se que existem poucos estudos e tecnologias desenvolvidas com a espécie A. denticulata e seus metabólitos secundários visando a obtenção de novas drogas anticâncer, tornando assim o cenário científico e tecnológico esperançoso para a realização de novos trabalhos e para o desenvolvimento de novas tecnologias para o tratamento do câncer, tendo em vista o vasto potencial que o gênero Aeschynomene apresenta relatado na literatura científica.

\section{Agradecimentos}

A Coordenação de Aperfeiçoamento de Pessoal de Nível Superior (CAPES) pelas bolsas de estudo.

\section{Referências}

A Baudino, T. (2015). Targeted cancer therapy: the next generation of cancer treatment. Current drug discovery technologies, 12(1), 3-20.

Abbas, Z., \& Rehman, S. (2018). An overview of cancer treatment modalities. Neoplasm, 1, 139-57.

Araujo Sousa, P. S., Nogueira, S. S., Ayala, K. N. R., Silva, P. C., da Silva Santos, E., de Sá, R. E., Lima Neto, F. E. M., Lima, J. R. C., Rodrigues, K. A. F, Rocha, J. A., \& Véras, L. M. C. (2021). Prospecção científica e tecnológica de Pilocarpus microphyllus e do alcaloide epiisopiloturina com ênfase na atividade antileishmania. Research, Society and Development, 10(7), e59810716984-e59810716984.

Araujo Sousa, P. S., Nogueira, S. S., Ayala, K. N. R., Silva, P. C., da Silva Santos, E., de Sá, R. E., Lima Neto, F. E. M., Lima, J. R. C., Rodrigues, K. A. F, Rocha, J. A., \& Véras, L. M. C. (2021). Prospecção científica e tecnológica de Pilocarpus microphyllus e do alcaloide epiisopiloturina com ênfase na atividade antileishmania. Research, Society and Development, 10(7), e59810716984-e59810716984.

Andricopulo, A. D., Salum, L. B., \& Abraham, D. J. (2009). Structure-based drug design strategies in medicinal chemistry. Current topics in medicinal chemistry, 9(9), 771-790.

EPO. (2019). WO2019030091A2 - Misturas herbicidas compreendendo lglufosinato e seu uso em culturas de algodão. https://worldwide.espacenet.com/patent/search/family/059579459/publication/WO2019030091A2?q=pn\%3DWO201903009A2

Fullas, F., Kornberg, L. J., Wani, M. C., Wall, M. E., Farnsworth, N. R., Chagwedera, T. E., \& Kinghorn, A. D. (1996). Two new aromatic constituents from the rootwood of Aeschynomene mimosifolia. Journal of natural products, 59(2), 190-192.

Galon, L., Guimarães, S., Radünz, A. L., Lima, A. M. D., Burg, G. M., Zandoná, R. R., Bastiani, N. O., Berlarmino, J. G., \& Perin, G. F. (2015). Relative competitiveness of irrigated rice cultivars and Aeschynomene denticulata. Bragantia, 74, 67-74.

Instituto Nacional De Câncer (INCA). (2017). ABC do câncer: abordagens básicas para o controle do câncer. 3. Rio de Janeiro.

Kirchhof, A. L. C., \& Lacerda, M. R. (2012). Retos y perspectivas de la publicación de artículos-un reflejo de los autores y editores. Texto \& ContextoEnfermagem, 21(1), 185-193.

Kissmann, K. G., \& Groth, D. (2000). Plantas infestantes e nocivas. BASF.

Leme, F. M., \& Scremin-Dias, E. (2014). Ecological interpretations of the leaf anatomy of amphibious species of Aeschynomene L.(LeguminosaePapilionoideae). Brazilian Journal of Biology, 74(1), 41-51.

Lima, D. F., Silva, R., Marques, L. G. A., Veras, L., Simões, E., de Almeida, J. R. D. S., Santos, M. R. M. C., \& Pessoa, C. (2015). Prospecção tecnológica do jaborandi (Pilocarpus microphyllus): espécie economicamente importante no norte e nordeste do Brasil. revista geintec-gestao inovacao e tecnologias, 5(1), 1626-1638. 
Maia, V. C., Catian, G., \& Leme, F. M. (2016). Neolasioptera pantaneira, a new species of Cecidomyiidae (Diptera) associated with Aeschynomene denticulata (Fabaceae) from Brazil. Brazilian Journal of Biology, 77, 170-175.

Mueller, S. P. M., \& Perucchi, V. (2014). Universidades e a produção de patentes: tópicos de interesse para o estudioso da informação tecnológica. Perspectivas em Ciência da Informação, 19, 15-36.

Newman, D. J., \& Cragg, G. M. (2016). Natural products as sources of new drugs from 1981 to 2014. Journal of natural products, 79(3), 629-661.

Nunes, F. E. A., Schaedler, C. E., \& Chiapinotto, D. M. (2018). Weed phytosociological survey in irrigated rice. Planta daninha, 36.

Rocha, C. A. M., Rabelo, N. L. F., Rodrigues, A. M., da Rocha, S. M., \& dos Reis, H. S. (2020). Prospecção Científica e Tecnológica do Ácido Caurenoico, um Diterpeno Bioativo. Cadernos de Prospecção, 13(1), 256.

Sakthivel, K. M., \& Guruvayoorappan, C. (2018). Targeted inhibition of tumor survival, metastasis and angiogenesis by Acacia ferruginea mediated regulation of VEGF, inflammatory mediators, cytokine profile and inhibition of transcription factor activation. Regulatory Toxicology and Pharmacology, 95 , 400-411.

Santos, L. B. D., Souza, J. K., Papassoni, B., Borges, D. G. L., Junior, G. A. D., Souza, J. M. E. D., Carollo, C. A., \& Borges, F. D. A. (2013). Efficacy of extracts from plants of the Brazilian Pantanal against Rhipicephalus (Boophilus) microplus. Revista brasileira de Parasitologia veterinária, 22, $532-538$.

Silva, H. R., dos Santos Nascimento, F. R., dos Santos, S. L., Lustosa, M. J. L., de Melo, J. C. L. C., Portela, C. L., Costa, R. H. F, Macedo Junior, C. A. A., Fernandes, L. K. S., \& Neto, J. C. P. (2020). A importância da prática de atividades físicas e uma alimentação saudável na profilaxia de um câncer. Research, Society and Development, 9(4), e68942868-e68942868.

Sharma, R., Rao, R., Kumar, S., Mahant, S., \& Khatkar, S. (2019). Therapeutic potential of citronella essential oil: a review. Current drug discovery technologies, 16(4), 330-339.

Tuttolomondo, T., La Bella, S., Licata, M., Virga, G., Leto, C., Saija, A., Trombetta, D., Tomaino, A., Speciale, A., Napoli, E. M., Siracusa, L., Pasquale, A., Curcuruto, G., \& Ruberto, G. (2013). Biomolecular characterization of wild sicilian oregano: phytochemical screening of essential oils and extracts, and evaluation of their antioxidant activities. Chemistry \& biodiversity, 10(3), 411-433.

Wei, A., \& Shibamoto, T. (2010). Antioxidant/lipoxygenase inhibitory activities and chemical compositions of selected essential oils. Journal of agricultural and food chemistry, 58(12), 7218-7225.

Weinberg, R. A. (2011). The Biology of Cancer. Garland Science. 